\title{
一类新型高苂光性类水滑石(Al-HTLc)的合成 与性能
}

章文贡 ${ }^{*}$, 陈鸿

福建师范大学化学与材料学院高分子研究所, 福州 350007

*联系人, E-mail: wgzang5678@tom.com

收稿日期: 2007-06-28; 接受日期: 2007-10-02

国家自然科学基金(批准号: 50272014)、福建省自然科学基金重大项目(批准号: 2001F005)和福建省纳米重大专项(批准号: 2005HZ01-5)资助

摘要采用共沉淀法, 合成、组装得到一类将铝离子 $\left(\mathrm{Al}^{3+}\right)$ 量减少到一定值的镁铝型类水 滑石, 并使分散于其镁铝水滑石板层上的铝离子与板层间溶于亲油性阴离子中的计量 8-着 基喹啉(8-HQ)配位, 获得一类新型高荧光性类水滑石(Al-HTLc). 采用荧光光谱、红外光谱、 紫外光谱、XRD、XPS 和热分析等对其进行了表征. 研究结果表明, 该 Al-HTLc 在紫外光

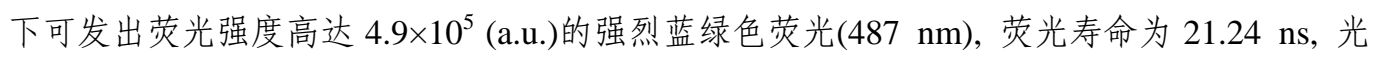
量子产率为 $67 \%$, 高于纯 8 -差基喹啉铝 $\left(\mathrm{Alq}_{3}\right)$, 且具有更高热稳定性, 有望作为一类高性能 的新型荧光材料而获得应用.
关键词

类水滑石

8-差基喹啉铝

插层

组装

超分子

荧光

荧光寿命

\section{1 前言}

类水滑石(hydrotalcite-like compounds, HTLc)系 一类由二价和三价金属离子组成的具有水滑石 (layered double hydroxides, LDHs) ${ }^{[1]}$ 层状晶体结构的 混合金属氢氧化物. 其组成通式为: $\left[\mathrm{M}(\mathrm{II})_{1-x} \mathrm{M}(\mathrm{III})_{x}\right.$ $\left.(\mathrm{OH})_{2}\right]^{x+}\left(\mathrm{A}^{n-}{ }_{x / n}\right) \cdot m \mathrm{H}_{2} \mathrm{O}$, 其中, $\mathrm{M}$ ( II ) 和 $\mathrm{M}(\mathrm{III})$ 分别为 二价和三价金属离子, $\mathrm{A}$ 是价数为 $-n$ 的层间阴离子, $x$ 为 HTLc中 $\mathrm{M}(\mathrm{III})$ 的摩尔数, $m$ 为水合水数. HTLc的层 状结构是由金属(氢)氧八面体籍共用边相互连接而成 的带剩余正电荷、厚度约 $1 \mathrm{~nm}$ 的板层, 层层之间具有 称为通道(gallery)的孔隙, 其中容纳有用于平衡电性 的可交换阴离子而使整个HTLc呈电中性, 阴离子和 HTLC片层间以静电引力和氢键相互作用. 虽然自然 界中天然水滑石物质只有一种, 但由于可形成HTLC 结构的二价和三价金属阳离子及层间阴离子较多, 因此人工合成的类水滑石种类繁多. 至今有关
HTLc的合成、结构、特性及其应用于离子交换 ${ }^{[2,3]}$ 、 吸附 ${ }^{[4]}$ 、分离 ${ }^{[5]}$ 、催化 ${ }^{[6,7]}$ 、医药 ${ }^{[8,9]}$ 等方面的研究相 当多, 其中功能性的HTLc的合成及应用已成为该领 域的研究热点之一.

众所周知, 具有良好的热稳定性, 宽激发带和高 荧光效率的 8-羊基喹啉铝 $\left(\mathrm{Alq}_{3}\right)$, 作为一种综合性能 优异的荧光金属配合物, 一直是被首选用于发光或 显示器件的荧光材料之一, 但其难以大面积成膜、成 本高等问题至今仍是困扰其进一步应用的难题，而 成为研究者关注的焦点.

基于以上研究背景, 本研究设想: 如果将镁铝水 滑石中铝离子 $\left(\mathrm{Al}^{3+}\right)$ 的含量降低至一适当值, 同时将 计量的 8-差基喹啉溶解或分散于镁铝水滑石层间的 硬脂酸根等亲油性阴离子中, 使其与均匀分布于水 滑石的氢氧化镁板层上的铝离子配位, 如此, 配体 (8-HQ)因与铝离子 $\left(\mathrm{Al}^{3+}\right)$ 的配位作用而 “悬挂”于氢氧 
化镁板层表面上，并且各单元被孤立固定于其上，从 而组装、形成一种完全不同于 8-羟基喹啉铝 $\left(\mathrm{Alq}_{3}\right)$ 的特殊配位结构, 由于该特殊结构具有比游离或 “自由”8-羊基喹啉铝更大的结构不对称性，因而可能 会提高其发光中心的荧光效率; 此外, 由于其中铝离 子是均匀分散、固定于板层上，因而可能会有效防止 各发光单元因团聚造成的荧光猝灭; 同时，由于该类 水滑石层状结构的保护作用，有可能提高该材料的 热稳定性. 本文即报告该类苂光性的类水滑石 (AlHTLc)的制备及其荧光光谱、红外光 谱、紫外光谱、 XRD、XPS 和热分析等表征的研究结果.

\section{2 实验部分}

\section{1 材料与试剂}

8-羊基喹啉(8-HQ)：国药集团化学试剂有限公司 (AR 分析纯); 其余试剂均为市售分析纯试剂.

\section{2 样品制备}

配制 $\mathrm{NaAlO}_{2}$ 水溶液(质量分数 $w=20 \%$ )0.12 g (A); 配制 $\mathrm{MgCl}_{2}$ 的水溶液 $(w=20 \%) 1.4 \mathrm{~g}(\mathrm{~B})$; 称取 8羟基喹啉 $0.13 \mathrm{~g}$, 用乙醇溶解后倒入 $3.37 \mathrm{~g}$ 硬脂酸钠 的水溶液 $(w=5 \%)$ 中 $(C)$. 在不停地强烈搅拌 $(C)$ 溶液 下，同时将溶液(A)和(B)逐滴加入(C)溶液中，用 $20 \%$ 氢氧化钠水溶液调节反应混合浆液至 $\mathrm{pH}=9$, 于 $80^{\circ} \mathrm{C}$ 下陈化 $12 \mathrm{~h}$, 抽滤、水洗涤滤饼至滤液 $\mathrm{pH}$ 为 7 8, 用 少量无水乙醇浸泡、洗涤沉淀物后抽滤, 滤饼于 $100{ }^{\circ} \mathrm{C}$ 下烘干 $3 \mathrm{~h}$, 得到荧光性类水滑石粉末样品 (Al-HTLc).

以 $0.4 \mathrm{~g} \mathrm{NaAlO}_{2}$ 水溶液(20\%)为(A)溶液, $3.37 \mathrm{~g}$ 蒸馏水为 $(C)$ 溶液, 重复以上操作, 制得用于比较的 $\operatorname{MgAl}-\mathrm{LDHs}(\mathrm{Mg}: \mathrm{Al}=6: 2)$ 粉末样品.

以 $0.12 \mathrm{~g} \mathrm{NaAlO}_{2}$ 水溶液(20\%)为(A)溶液, $3.37 \mathrm{~g}$ 蒸馏水为 $(C)$ 溶液, 重复以上操作, 制得用于比较的 MgAl-LDHs ( $\mathrm{Mg}: \mathrm{Al}=6: 0.6$ ) 粉末样品.

以 $0.12 \mathrm{~g} \mathrm{NaAlO}_{2}$ 水溶液(20\%)为(A)溶液, $3.37 \mathrm{~g}$ 硬脂酸钠的水溶液 $(5 \%)$ 为 $(\mathrm{C})$ 溶液, 重复以上操作, 制得用于比较的 MgAl-硬脂酸根-LDHs ( $\mathrm{Mg}: \mathrm{Al}=$ $6: 0.6)$.

\section{3 表征}

XRD: 用荷兰 Philips 公司 XPert MPD 型 X 射线 粉末衍射仪 $(\mathrm{Cu}$ 靶, $\mathrm{K} \alpha$ 射线, $\lambda$ 为 $0.15418 \mathrm{~nm}, 40 \mathrm{kV}$, $40 \mathrm{~mA}$, 扫描速度 $3^{\circ} / \mathrm{min}$, 角度范围 $\left.3^{\circ} \sim 70^{\circ}\right)$ 测定.

红外光谱：用美国 Thermo Nicolet 公司 AVATAR360 型傅里叶红外光谱仪(分辨率 $4 \mathrm{~cm}^{-1}$, 扫 描次数 64 , 样品: $\mathrm{KBr}=1: 100$, 质量比).

可见-紫外光谱：用美国 Perkin Elmer 公司 Lambda900 型紫外-可见分光光度计, 光谱扫描速度 为快速, 狭缝宽 $2.0 \mathrm{~nm}$.

荧光光谱: 用英国 Edinburgh 公司 FL/FS 920 TCSPC 型稳态/瞬态苂光分光光度计, 稳态苂光光谱 测定时所有狭缝宽度固定在 $0.5 \mathrm{~nm}$.

热稳定性：用德国 NETZSCH STA 449C 型热分 析仪(升温速率 $10 \mathrm{~K} / \mathrm{min}$, 空气气氛).

XPS: 用 PHI Quantum 2000 Scanning ESCA Microprobe, 采用 CAE 模式, 分析器通过能为: (1) 宽扫 描谱(su) $E_{\mathrm{p}}=187.85 \mathrm{eV}$; (2) 窄扫描谱(mu)(A)通常收 谱条件时 $E_{\mathrm{p}}=29.35,58.70$ 或 $117.40 \mathrm{eV} . \mathrm{X}$ 射线源: 单

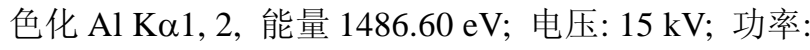
$35 \mathrm{~W}$; 束斑直径: $200 \mu \mathrm{m}$. 光电子出射角 $45^{\circ}$. 仪器分 析室基础真空: $5 \times 10^{-8} \mathrm{~Pa}$, XPS 收谱时真空为: $5 \times 10^{-7}$ Pa.

\section{3 结果与讨论}

\section{1 红外光谱分析}

MgAl-LDHs(Mg:Al = 6:2)、8-羟基喹啉和 Al-HTLc试样的FT-IR谱图如图 1(a), (b), (c)所示. 可 以看出: (1) MgAl-LDHs在 $1637 \mathrm{~cm}^{-1}$ 和 $3455 \mathrm{~cm}^{-1}$ 附 近的吸收峰分别对应于水滑石层板的羟基或物理吸 附水分子的 $v_{\mathrm{H}-\mathrm{O}-\mathrm{H}}$ 弯曲振动和 $v_{\mathrm{OH}}$ 对称收缩振动 ${ }^{[11]}$. 表 明样品颗粒表面吸附或晶格层间插入相当数量的 $\mathrm{H}_{2} \mathrm{O}$ 分子, 相对于自由羟基 $\left(3650 \mathrm{~cm}^{-1}\right), v_{\mathrm{OH}}$ 对称收缩 振动有较小值, 表明水滑石层板和层间所有的羟基 均以氢键相连 ${ }^{[12]}, 450 \mathrm{~cm}^{-1}$ 附近的红外吸收来源于板 层表面的羟基, $1381 \mathrm{~cm}^{-1}$ 处的吸收由层间 $\mathrm{C}^{-}$通过层 板差基与 $\mathrm{Mg}$ 原子配位的 $\mathrm{C} 1-\mathrm{HO}-\mathrm{Mg}$ 骨架振动引起; (2) Al-HTLc的FT-IR谱图(c)中, 在 2921, $2852 \mathrm{~cm}^{-1}$ 处出 现明显的甲基、亚甲基的特征吸收峰，在 1465 


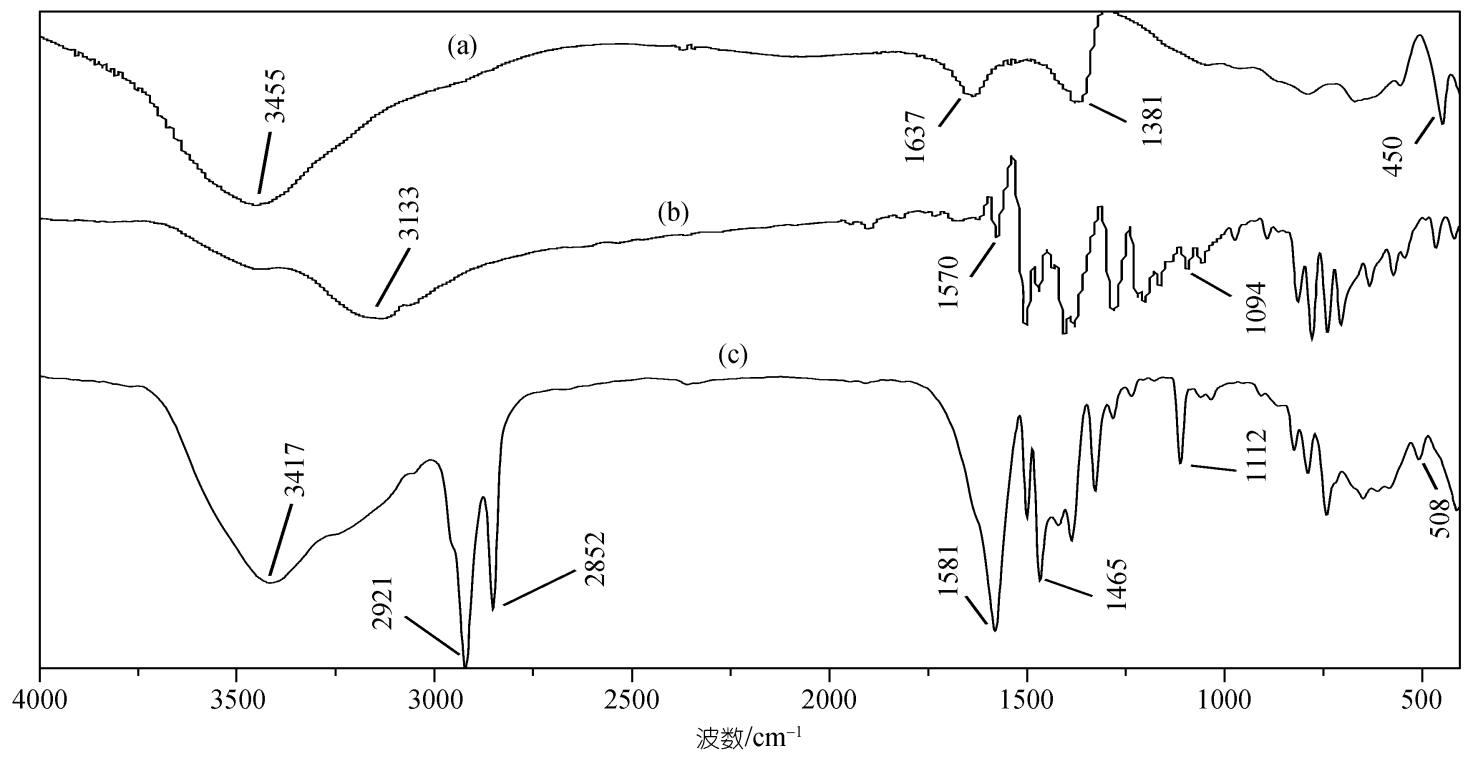

图 $1 \mathrm{MgAl}-\mathrm{LDHs}(\mathrm{Mg}$ : Al = 6:2)(a), 8-羟基喹啉(b)和 Al-HTLc(c)试样的 FT-IR 谱图

$\mathrm{cm}^{-1}$ 出现了羧酸根离子的不对称吸收谱带，说明硬 脂酸根离子已进入水滑石层间; (3) 对比(b)和(c)的谱 图可以看到, 配体 8-羟基喹啉在 $3133 \mathrm{~cm}^{-1}$ 处的 $\mathrm{O}-\mathrm{H}$ 伸缩振动吸收峰在(c)谱图中消失，表明层间 8-差基 喹啉上的酚羟基去质子化后与层板上的 $\mathrm{A} 1^{3+}$ 离子发 生了配位; 8-差基喹啉(图(b))中位于 $1570 \mathrm{~cm}^{-1}$ 处的吡 啶环的 $\mathrm{C}-\mathrm{N}$ 伸缩振动频率, 红移至 $1581 \mathrm{~cm}^{-1}$ 处, 说 明氮原子参与了成键 ${ }^{[13]}$. 8-差基喹啉在 $1094 \mathrm{~cm}^{-1}$ 处 的位于酚环上的 $\mathrm{C}-\mathrm{O}$ 的伸缩振动频率，红移至 1112 $\mathrm{cm}^{-1}$ 处, 此峰是 8-差基喹啉与 $\mathrm{Al}^{3+}$ 离子配位后生成的 $\mathrm{C}-\mathrm{O}-\mathrm{Al}$ 键中的 $\mathrm{C}-\mathrm{O}$ 伸缩振动产生的 ${ }^{[14,15]}$. (c) 谱 图 $508 \mathrm{~cm}^{-1}$ 处为 $\mathrm{Al}-\mathrm{O}$ 伸缩振动频率, $414 \mathrm{~cm}^{-1}$ 处为 $\mathrm{Al}-\mathrm{N}$ 伸缩振动频率. 由此可以证明, Al-HTLc中位于 类水滑石层间的 8-羟基喹啉配体已经通过其分子上 的酚羟基以及氮原子与层板上的铝离子配位形成了 配合物.

\section{2 紫外-可见吸收光谱(UV/Vis)}

Al-HTLc 和 8-羊基喹啉试样的 UV/Vis 谱图如图 2 所示. 从图上可以看出, 自由配体 8-差基喹啉分别 在 324, $222 \mathrm{~nm}$ 出现两个吸收峰. $222 \mathrm{~nm}$ 处的短波吸 收峰来自苯环的 $\pi-\pi *$ 电子跃迁, $324 \mathrm{~nm}$ 处的吸收峰来 自自由配体中喹啉环所产生的 $n-\pi *$ 跃迁的吸收带. 当 $\mathrm{Al}^{3+}$ 离子与配体配位后, $\mathrm{N}$ 原子上的孤电子对与金

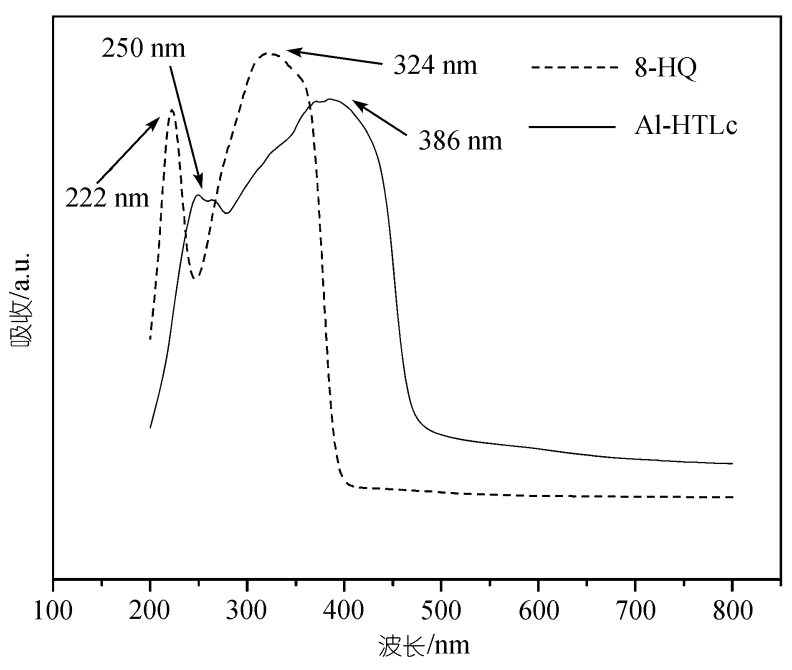

图 2 Al-HTLc 和 HQ 试样的 UV/Vis 谱图

属离子空轨道相互交叠, 使 $\mathrm{n}$ 轨道能量下降, 同时由 于电子云交叠也增加了 $\pi$ 轨道电子的离域范围, 使体 系更稳定, 因而两个吸收峰都向长波方向移动. 这是 8-差基喹啉配体与 $\mathrm{Al}^{3+}$ 形成配位键的有力证据 ${ }^{[16]}$.

\section{3 苂光光谱}

\subsection{1 苂光强度}

按文献[17]制备 8-差基喹啉铝( $\mathrm{Alq}_{3}$ )与 Al-HTLc 进 行荧光性质的比较，如图 3 所示. 由图可见，图 3(a), 


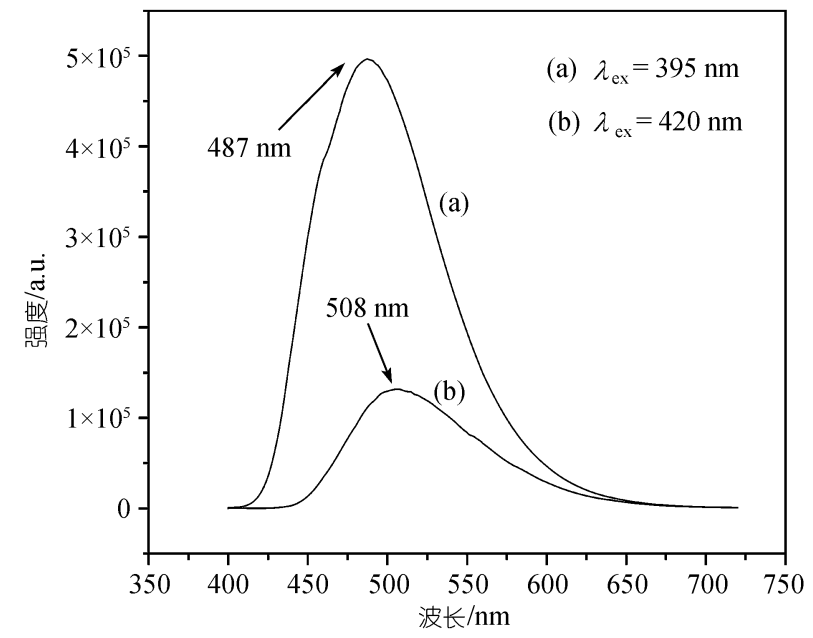

图 3 Al-HTLc(a)和 $\mathrm{Alq}_{3}(\mathrm{~b})$ 试样的苂光光谱

(b)均在 420 642 $\mathrm{nm}$ 之间出现一个较大的荧光发射峰, 介于蓝光与绿光之间, 发光机理属于金属离子微扰 配体的发光, 即 $\mathrm{L}^{*}-\mathrm{L}$ 型发光, 是由电子在配体中的 $\pi-\pi *$ 能级之间跃迁产生的. 但 $\mathrm{Alq}_{3}$ 的峰值在 $508 \mathrm{~nm}$, 而 Al-HTLc 的峰值在 $487 \mathrm{~nm}$, 蓝移了 $21 \mathrm{~nm}$, 这主要 是由于 8-羟基喹啉铝配位结构在水滑石中配位后, 分子间的相互作用增加以及相邻的两个发光单元产 生的激子减少所导致. 另外, $\mathrm{Alq}_{3}$ 的荧光强度仅有 $1.3 \times 10^{5}$ (a.u.), 而 Al-HTLc 的苂光强度达 $4.9 \times 10^{5}$ (a.u.), 强度明显增强.

可能的原因是由于在 $\mathrm{Al}-\mathrm{HTLC}$ 中, $\mathrm{Al}^{3+}$ 是较均匀 地镶嵌于水滑石层板中, 而与之配位的 8-羟基喹啉 存在于层间, 受空间和层板的阻隔, 使得 8-羊基喹啉

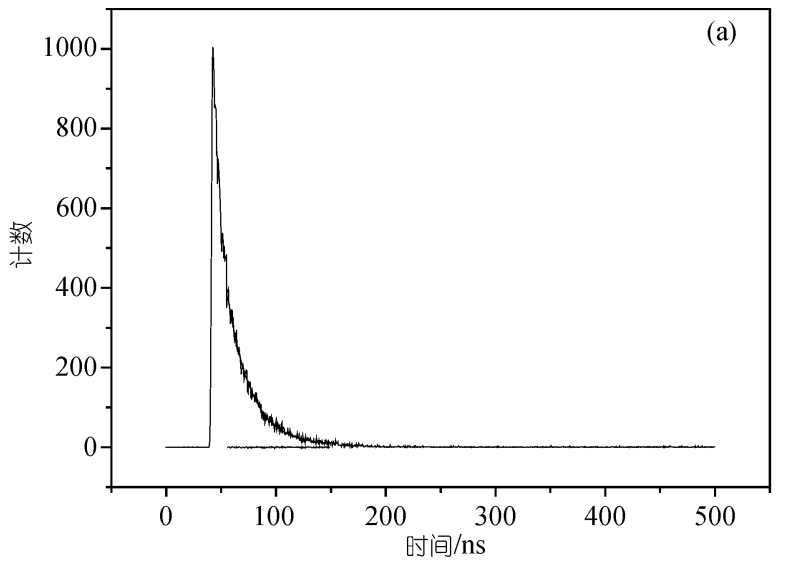

铝发光结构单元难于接近、聚集，所以不易产生荧光 猝灭; 溶于层间阴离子中的配体与位于板层上的 $\mathrm{Al}^{3+}$ 配位，其所形成的特殊组装结构会提高发光中心的 不对称性, 因而 Al-HTLc 能发出较强苂光. 也就是说, 在配体 8-羟基喹啉含量显著降低的情况下, Al-HTLc 能发出比相应纯配合物更强的荧光.

\subsection{2 苂光寿命}

本实验用氢气纳秒闪秌灯来激发Al-HTLc样品, 测量其荧光寿命, 激发脉冲重复频率为 $40000 \mathrm{~Hz}$, 中 心波长为 $368 \mathrm{~nm}$. 通过进行双指数过程拟合, 所得荧 光寿命拟合曲线如图 4(a)所示，样品的荧光寿命为 21.24 ns. 在同样试验条件下测得纯 $\mathrm{Alq}_{3}$ 的荧光寿命 是 $13.92 \mathrm{~ns}$, 说明Al-HTLc样品的荧光寿命比纯 $\mathrm{Alq}_{3}$ 有所延长. 分析其主要原因是：根据荧光寿命 ${ }^{[18]}$ 的 定义:

$$
\tau=1 /\left(k_{\mathrm{f}}+\Sigma K\right),
$$

其中, $k_{\mathrm{f}}$ 代表苂光发射的速率常数, $\Sigma K$ 代表各种单分 子的非辐射去活化过程的速率常数的总和. 由于 Al-HTLc样品的发光单元是处在水滑石层状固体基 质中, 增加了其结构的刚性, 随着刚性结构的增强, 其荧光发射速率会增大 ${ }^{[18]}$ ，即增大了 $k_{\mathrm{f}}$; 然而，在 $\mathrm{Al}-\mathrm{HTLC}$ 中, 由于分散于水滑石板层中的 $\mathrm{Al}^{3+}$, 与溶 于层间阴离子中的 8-羟基喹啉形成了 “挂在” 板层表 面上的特殊配位结构，使发光单元之间以及发光单 元与环境之间碰撞几率减少，而这种碰撞作用是发 光中心非辐射跃迁的主要途径，再加之发光单元的

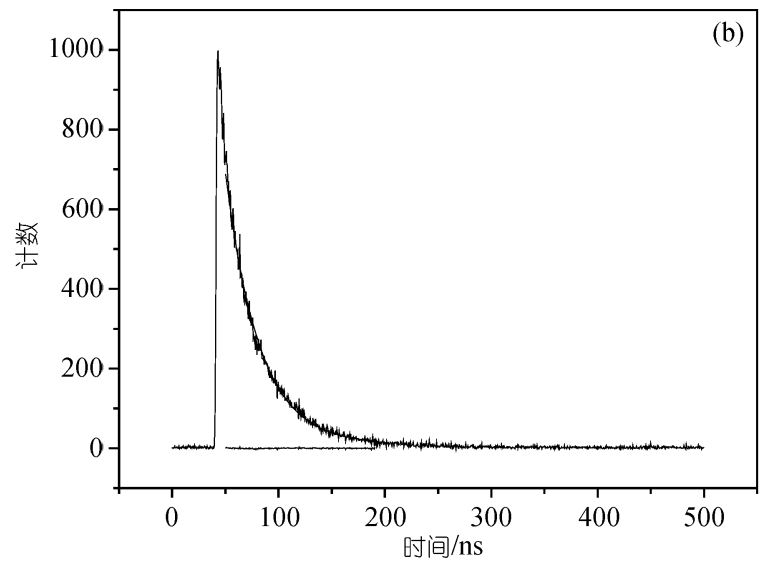

图 4 Al-HTLc 试样的荧光寿命谱

(a) $293.15 \mathrm{~K}$; (b) $10 \mathrm{~K}$ 
结构刚性增强，导致其键振动减弱，这都导致发光中 心非辐射跃迁减少, 即 $\Sigma K$ 变小, $\Sigma K$ 减小的趋势比 $k_{\mathrm{f}}$ 的增大趋势大，因此其荧光寿命变长.

为了进一步比较Al-HTLc与 $\mathrm{Alq}_{3}$ 的发光能力, 按 照荧光量子产率和荧光寿命之间的关系 ${ }^{[18]}$, 即: $Y_{\mathrm{f}}=$ $\tau / \tau_{0}$. 以低温 $(10 \mathrm{~K})$ 下的寿命来代替本征寿命 $\tau_{0}$, 根据 图 4(b)所示实测的荧光寿命拟合曲线 $\left(\tau_{0}=31.69 \mathrm{~ns}\right)$ 进 行计算，测得Al-HTLc荧光量子产率为 $67 \%$, 而于同 条件下测得纯 $\mathrm{Alq}_{3}$ 的苂光量子产率为 $57 \%$. 可见, Al-HTLc的苂光量子产率比纯 $\mathrm{Alq}_{3}$ 高了 $10 \%$.

\section{$3.4 \mathrm{XRD}$ 分析}

图 5 为 $\mathrm{MgAl}-\mathrm{LDHs}(\mathrm{Mg}: \mathrm{Al}=6: 2), \mathrm{MgAl}-\mathrm{LDHs}$ $(\mathrm{Mg}: \mathrm{Al}=6: 0.6), \mathrm{MgAl}$-硬脂酸根-LDHs和Al-HTLc试 样的XRD图谱, 可以看出, MgAl-LDHs $(\mathrm{Mg}: \mathrm{Al}=$ 6:2), MgAl-LDHs ( $\mathrm{Mg}: \mathrm{Al}=6: 0.6)$, MgAl-硬脂酸根LDHs均具有水滑石的完整层状晶体结构特征 ${ }^{[1]}$, 其 反映层状结构的衍射强度最大的三个特征衍射峰 $(2 \theta)$ 位置出现在 $11^{\circ}, 23^{\circ}$ 和 $34^{\circ}$ 附近, 分别对应着层间 距 $d$ (003)和两个高级反射 $d$ (006), $d$ (009). 除(003), (006), (009), (015), (018), (110)外，没有发现其他衍射 峰, 基线低且平稳, 主要衍射峰峰型尖锐. 对比MgAlLDHs(Mg:Al = 6:2)和MgAl-LDHs( $\mathrm{Mg}: \mathrm{Al}=6: 0.6)$, 衍射峰位置基本不变, 只是强度有所改变, 可见 $\mathrm{Al}$ 与 $\mathrm{Mg}$ 的比例发生变化, 并未对产物的层状晶体结构造 成影响; 在 MgAl-硬脂酸根-LDHs中, 三个特征衍射 峰变宽且强度降低, 并向低角度移动, 层间距变大. 根据布拉格方程 $(2 d \sin \theta=\lambda)$, 可知层间距由原来的 $0.76 \mathrm{~nm}$ 变为 $0.82 \mathrm{~nm}$, 说明硬脂酸根已插入层间, 结 晶度降低. 另外, 在 $2 \theta$ 为 $60^{\circ}$ 附近的(110)衍射峰的位 置基本没有变化, (110)衍射峰的位置代表层板上原子 的排列密度, 这说明 $\mathrm{Al}$ 与 $\mathrm{Mg}$ 的比例的改变, 硬脂酸 根的加入, 仅使层间距发生了变化, 对层板的结构没 有影响; 在Al-HTLc中, 归属于(003)面的特征衍射峰 不见了, 造成此峰不见的原因可能是移向更低的 $2 \theta$ 角度, 或确已消失. 而且, 其余的高级衍射峰也变弱 或消失, 并伴有很多的杂峰(杂峰来源于 8-差基喹啉), 这表明加入配体 8-差基喹啉后，进入层间的量的增 加, 层板被撑得更开, Al-HTLc趋向形成具有更大层 间距的低晶化、无定形甚至是剥离的类水滑石产物.

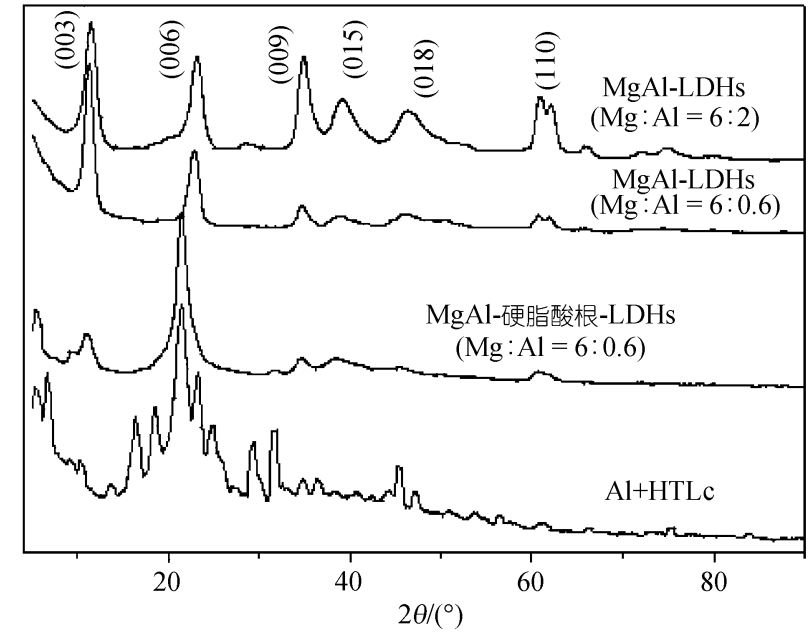

图 $5 \mathrm{MgAl}-\mathrm{LDHs}(\mathrm{Mg}: \mathrm{Al}=6: 2) 、 \mathrm{MgAl}-\mathrm{LDHs}(\mathrm{Mg}: \mathrm{Al}=$ 6:0.6)、MgAl-硬脂酸根-LDHS 和 Al-HTLc 试样的 XRD 谱图

XRD 表征结果说明 8-着基喹啉确已进入水滑石层间.

\subsection{XPS 分析}

图 6 为 $\mathrm{Alq}_{3}$ 试样表面的 XPS 全扫描谱, 其中 5 个明显的峰: 531.12, 399.37, 284.87, 118.7 和 $74.08 \mathrm{eV}$, 分别对应于 $\mathrm{O} 1 \mathrm{~s}, \mathrm{Nls}, \mathrm{Cls}, \mathrm{A} 12 \mathrm{~s}$ 和 A12p 的电子态. 图 7 分别给出了 Al-HTLc 和 $\mathrm{Alq}_{3}$ 试样的 A12p, Cls, Nls 和 O1s 的精细谱. 由图可见, Al-HTLc 中 A12p, Cls, Nls, O1s 的结合能都发生了改变. Cls 向低结合能方向移 动了 $0.3 \mathrm{eV}$, 强度有所增加, $\mathrm{O} 1 \mathrm{~s}$ 也向低结合能方向移 动了 $0.4 \mathrm{eV}$, 强度减弱, 反映出 $\mathrm{C}, \mathrm{O}$ 原子的周围环境 发生了改变, 影响了原子周围的电子云的屏蔽效应,

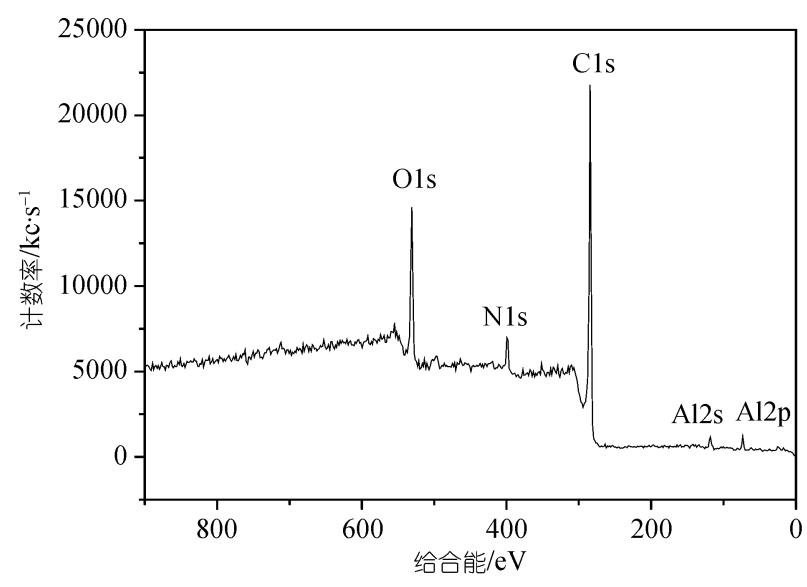

图 $6 \mathrm{Alq}_{3}$ 试样的 XPS 全扫描谱 

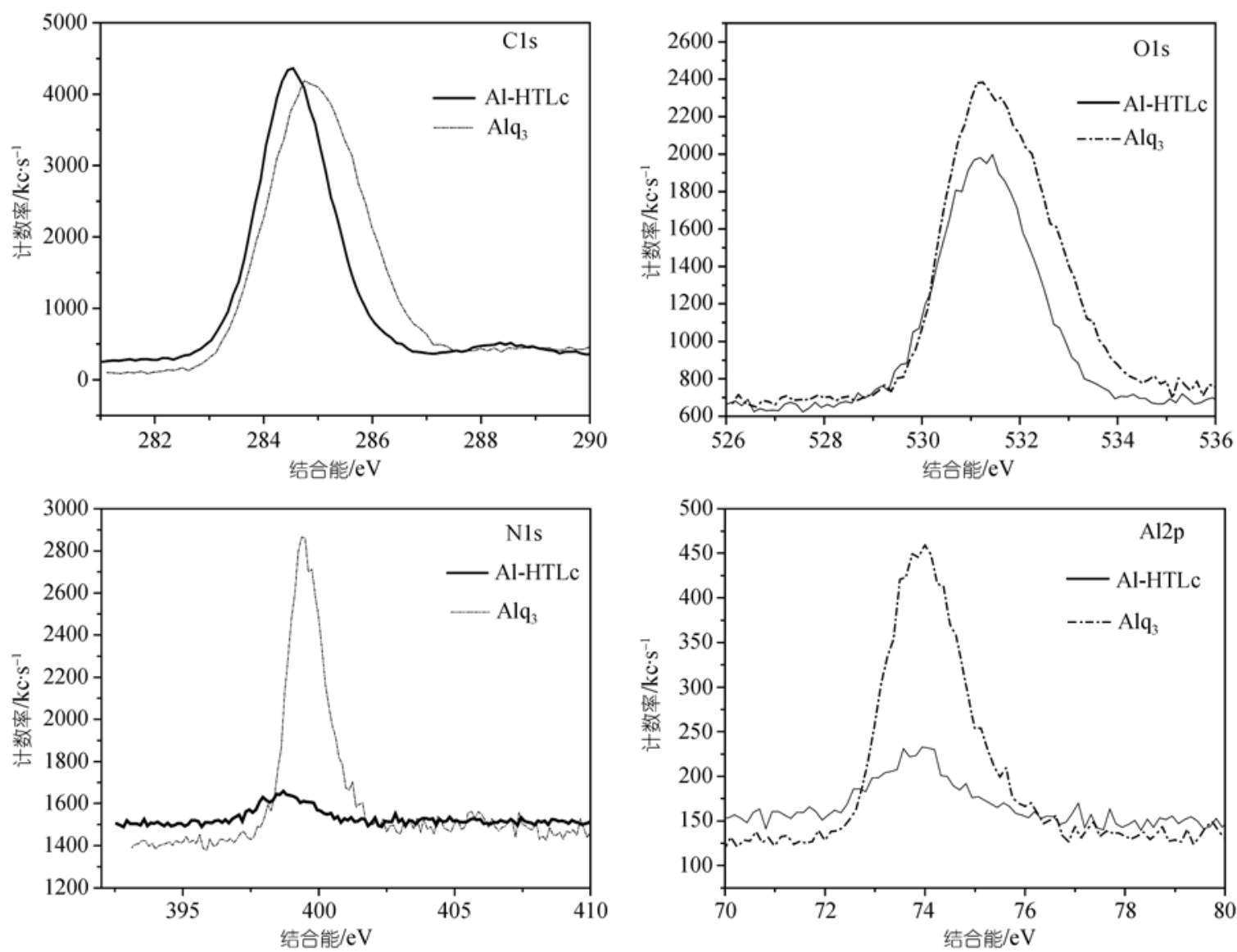

图 $7 \mathrm{Alq}_{3}$ 和 Al-HTLc 试样的 C1s, O1s, N1s, Al2p 精细谱

使结合能发生改变; A12p 和 Nls 的谱峰变弱,变宽, 变 得不规则, 并向低结合能方向移动. 峰面积减少, 说 明原子的相对含量降低. 结合能下降, 说明 Al-HTLC 中 $\mathrm{Al}$ 和 $\mathrm{N}$ 之间的相互作用比在 $\mathrm{Alq}_{3}$ 中更为强烈, $\mathrm{N}$ 的电子更多地转移到 $\mathrm{Al}^{3+}$ 的外层空轨道上, 使其外层 电荷密度增加, 内层电子结合能下降. 以上分析说明, $\mathrm{Al}-\mathrm{HTLC}$ 分子中 $\mathrm{C}, \mathrm{O}, \mathrm{N}$ 和 $\mathrm{Al}$ 原子的周围环境不同于 纯 $\mathrm{Alq}_{3}$, 即 Al-HTLc 中 8-着基喹啉铝配位结构与纯 $\mathrm{Alq}_{3}$ 的分子结构不同, 已不再是 8-差基喹啉环绕在中 心 $\mathrm{Al}^{3+}$ 周围的, 这也间接证实了 8-羟基喹啉已插入水 滑石层间。

\section{6 热稳定性分析}

图 8(a), (b)分别是 MgAl-LDHs 试样及 Al-HTLc 试样的 TG-DSC 图谱, 从图 8(a)TG 曲线可见, $\mathrm{MgAl}-\mathrm{LDHs}$ 在室温到 $215^{\circ} \mathrm{C}$ 之间有一失重峰, 失重率 为 $12 \%$, 主要是表面吸附水和层间水的脱除, 这一过
程通常是可逆的; 在 $350^{\circ} \mathrm{C} 448^{\circ} \mathrm{C}$ 之间迅速地失重, 失重率为 $20 \%$, 对应DSC 曲线出现非常明显的吸热 峰, 峰值在 $410^{\circ} \mathrm{C}$, 主要是层板羟基和层间阴离子脱 除，标志着层状结构的破坏 ${ }^{[19]}$.

在图 8(b)TG 谱线中, 从室温到 $313^{\circ} \mathrm{C}$ 之间失重 率仅为 3\%, 主要是物理水的脱除; 在 313 553 ${ }^{\circ} \mathrm{C}$ 之间 出现迅速且大量地失重, 失重率达 $80 \%$, 对应 DSC 曲 线出现一个非常明显的放热峰, 峰值在 $531^{\circ} \mathrm{C}$, 这主 要是层间有机物(硬脂酸根离子和 8-差基喹啉)在空气 中氧化(燃烧)以及层板羟基的脱除产生的. $553^{\circ} \mathrm{C}$ 以 上分解的残余物是镁、铝的氧化物, 此时重量和热重 趋于平衡.

比较发现，由于 8-着基喹啉铝配合物与主体层 板之间存在相互作用, Al-HTLc 的热失重高峰较 MgAl-LDHs 向高温方向移动, 说明该试样的热稳定 性比水滑石有所提高. 

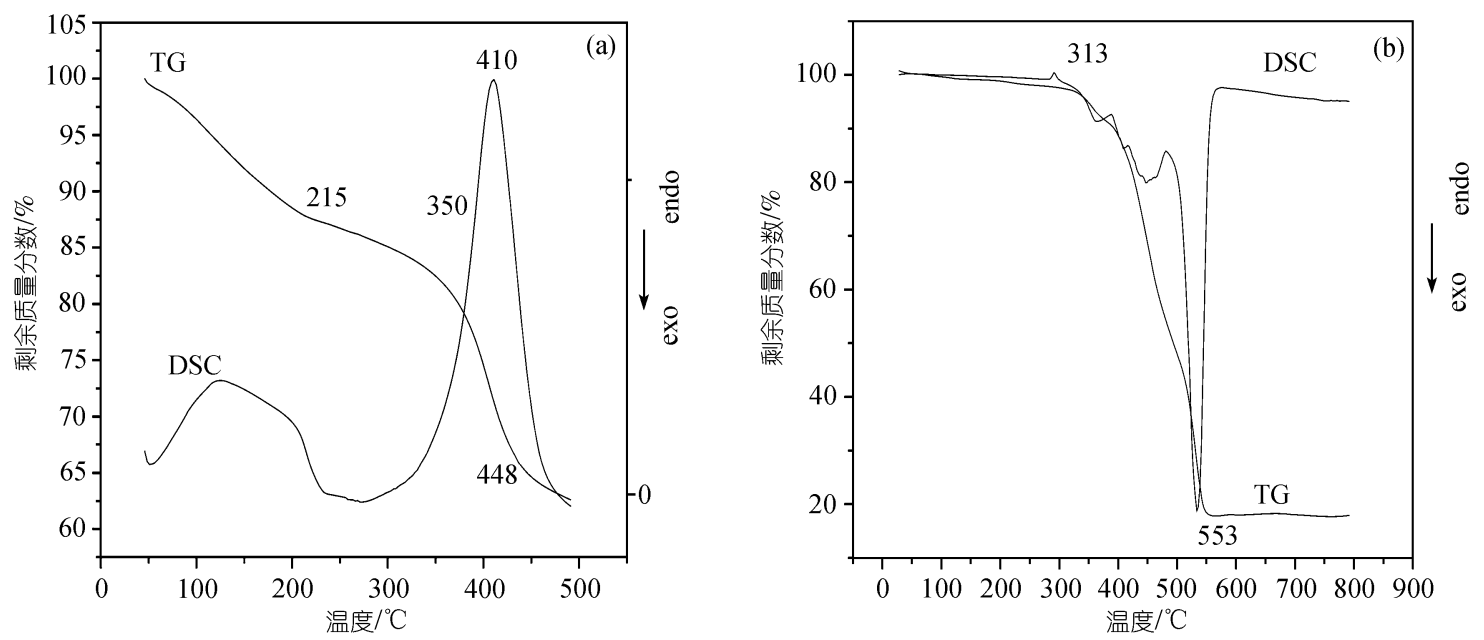

图 8 MgAl-LDHs (a)和 Al-HTLc (b)试样的 TG-DSC 谱

根据文献[20], 8-羊基喹啉的熔点为 $75 \sim 76^{\circ} \mathrm{C}$ (分 解), 受潮变黄, 固体及溶液露置于空气中会变黄进 而变黑, $\mathrm{Alq}_{3}$ 的溶度积常数 $K_{\mathrm{sp}}=1.0 \times 10^{-29}$, 热稳定性 为 $102 \sim 220^{\circ} \mathrm{C}$. 而本实验的 Al-HTLc 试样, 在 $313^{\circ} \mathrm{C}$ 才开始分解, 热稳定性明显高于 $\mathrm{Alq}_{3}$, 这可能是由于 水滑石层状结构 “笼” 的保护作用所致.

\section{4 结论}

本研究采用共沉淀法, 合成、组装得到一类将铝 离子 $\left(\mathrm{Al}^{3+}\right)$ 量减少到一定值的镁铝型类水滑石, 并使
分散于其镁铝水滑石板层上的铝离子与板层间溶于 亲油性阴离子中的计量 8-差基喹啉配位，获得一类 新型高苂光性类水滑石(Al-HTLc). 采用苂光光谱、红 外光谱、紫外光谱、XRD、XPS 和热分析等对其进 行了表征. 研究结果表明, 该 Al-HTLc 在紫外光下可 发出苂光强度高达 $4.9 \times 10^{5}$ (a.u.) 的强烈蓝绿色苂光 (487 nm), 荧光寿命为 $21.24 \mathrm{~ns}$, 光量子产率为 $67 \%$, 高于纯 8-羊基喹啉铝 $\left(\mathrm{Alq}_{3}\right)$, 且具有更高热稳定性, 有望作为一类高性能的新型荧光材料而获得应用.

\section{参考文献}

1 Canavi F, Trifiro F, Vaccari A. Hydrotalcite-type anionic clays: Preparation, properties and applications. Catal Today, 1991, 11: 173301 [DOI]

2 邢渘, 李殿卿, 任玲玲, Evans D G, 段雪. 超分子结构水杨酸根插层水滑石的组装及结构与性能研究. 化学学报, 2003, 61(2): $267-272$

3 苟国敬, 马培华, 褚敏雄. 四硼酸根阴离子对氯型水滑石前体插层组装的动力学研究. 高等学校化学学报, 2005, 26: 497一 502

4 Latterini L, Nocchetti M, Aloisi G G, Costantino U, Elisei F. Organized chromophores in layered inorganic matrices. Inor Chim Acta, 2006, 7: 48-61

5 Bhaumik A, Samanta S, Mal N K. Efficient removal of arsenic from polluted ground water by using a layered double hydroxide exchanger. Indian J Chem Section $\alpha$-Inorga Bio-Inorg Phys Theor Anal Chem, 2005, 44(7): 1406-1409

6 杨飘萍，吴通好。水滑石的结构性质、合成及催化应用。石化技术与应用, 2005, 23(1): 61-66

7 Prescott H A, Li Z J, Kemnitz E. Application of calcined Mg-Al hydrotalcites for Michael additions: An investigation of catalytic activity-and acid-base properties. J Catal, 2005, 234(1): 119-130[DOI]

8 Wei M, Xu X Y, Wang X R, Li F, Zhang H, Lu Y L, Pu M, David G. Evans, Duan X. Study on the photochromism of Ni-Al layered double hydroxides containing nitrate anions. Eur J Inorg Chem, 2006, 14: 2831-2838[DOI]

9 You Y W, Zhao H T, Vance G F. Hybrid organic-inorganic derivatives of layered double hydroxides and dodecylbenzenesulfonate: 
Preparation and adsorption characteristics. J Mater Chem, 2002, 12: 907-912 [DOI]

10 Tang C W, Vanslyke S A. Organic electroluminescence diodes. Appl Phys Lett, 1987, 51(12): 913 — 915[DOI]

11 Mohd Zobir bin Hussein, Tan K H. Synthesis and properties of layered organic-inorganic hybrid material: Zn-Al layered double hydroxide-dioctyl sulfosuccinate nanocomposite. J Nanopart Rese, 2000, 2: 293-298[DOI]

12 Velu S, Ramkumar V, Narayanan A. Effect of interlayer anions on the physicochemical properties of zinc-aluminum hydrotalcite-like compounds. J Mater Sci, 1997, 32: 957-964[DOI]

13 王崇侠, 余忠清, 李邮, 王炳祥, 黄晓华. 二(2-苯基-8-差基喹啉)锌和喹啉锌的合成和荧光性质. 发光学报, 2004, 25(4): 414-418

14 郝玉英, 郝海涛, 王华, 周禾丰, 刘旭光, 许并社. 2(8-差基喹啉)-2(苯酚)合锆薄膜的制备与性能研究. 光谱学与光谱分析, 2004 , 24(12): 1524-1527

15 马礼敦. 高等结构分析. 上海: 复旦大学出版社, 2002. 157-165

16 欧阳新华, 曾和平, 谢彦.8-着基喹啉衍生物及其金属配合物的合成与光致发光特性. 有机化学, 2007, 27(3): 402-408

17 朱凌健, 郭灿城. 高纯 8-差基喹啉铝的简便合成方法. 化学试剂, 2004, 26(6): 369-370

18 陈国珍, 黄贤智, 郑朱梓. 荧光分析法. 第 2 版. 北京: 科学出版社, 1990. 15-17

19 Qiu L Z, Qu B J. Preparation and characterization of surfactant-free polystyrene/layered double hydroxide exfoliated nanocomposite via soap-free emulsion polymerization. J Collo Interf Sci , 2006, 301: 347-351[DOI]

20 邹永德，黄新邦，林位株. 8-差基喹啉铝高分子薄膜的荧光特性. 物理化学学报, 1999, 15(4): 375-380 